\title{
Simulation and analysis on low-frequency scattering characteristics of the finite cylindrical shell in shallow water
}

\author{
Jinyu $\mathrm{Li}^{1,2,3}$, Dejiang Shang ${ }^{1,2,3}$, and Yan Xiao ${ }^{1,2,3, *}$ \\ ${ }^{1}$ Acoustic Science and Technology Laboratory, Harbin Engineering University, Harbin 150001, China \\ ${ }^{2}$ Key Laboratory of Marine Information Acquisition and Security (Harbin Engineering University), Ministry of Industry and \\ Information Technology; Harbin 150001, China \\ ${ }^{3}$ College of Underwater Acoustic Engineering, Harbin Engineering University, Harbin 150001, China
}

\begin{abstract}
Low-frequency acoustic scatterings from a finite cylindrical shell are numerically analyzed by FEM. The simulation results show that the acoustic-scattering field in waveguide has lots of frequency-related sidelobes, while no sidelobes exist in free space at low frequencies. The simulation also indicates that the module value in waveguide can be almost $20 \mathrm{~dB}$ larger than that in free space at low frequency, which is caused by the ocean boundaries. We also demonstrate that when the incident wave direction is normal to the target at low frequency, the target strength will be maximum and the distribution of the acoustic-scattering field is axisymmetric about the incident waving direction. Meanwhile, the acoustic-scattering field is also related to the impedance of the seabed, and the change of the impedance makes just a little contribution to the scattering field. Finally, the influence of different target locations is analyzed, including the targets near the sea surface, seabed and the middle region of the ocean waveguide, respectively. From simulation results, it is evident that the distribution of the acoustic-scattering field at low frequency has a little difference, which is smaller than $0.5 \mathrm{~dB}$ with various target locations, and the change is frequency and boundary-related.
\end{abstract}

\section{Introduction}

Scattering has always been important issues in the history of underwater acoustic engineering. In the past, Ingenito[1] used Eigen Function to study the scattering field of rigid sphere in layered waveguide; Gary S. Sammelmann and Roger H. Hackman studied the acoustic scattering field from an elastic spherical shell in a homogeneous, range-independent waveguide[3]. Several methods, such as PE, theory of normal mode, theory of ray, FEM, BEM, and so on, have been developed for solving problems in both underwater propagation[4] and free-space scattering[5].

Considering the irregularity of realistic target shape, this paper uses the method of finite element to calculate the sound scattering field, just to discover some regular laws of the object scattering in the waveguide at low frequency. The work of this paper is contributed to further study on theory of scattering at low frequency in the waveguide.

\section{Theory}

Since the shape of realistic objects may not be regular, this paper uses the method of finite element to calculate the scattering field of the target, combining the theory of the fluid-structure interaction with boundaries conditions.

\subsection{FEM}

The basis of the FEM[6] is the division of the physical domains into subdomains, or elements, within which exact or approximate solutions can be obtained in terms of a finite number of degrees-of-freedom. The connectivity between the elements then leads to a linear system of equations in the degrees-of-freedom, closely analogous to the system of equations describing the mechanical behaviour of discrete systems. It is a general numerical solution technique for field and boundaryvalue problems.

An acoustic medium with space-varying sound speed and density occupies the volume, bounded by the boundary. In addition, the medium is assumed to be a viscous fluid without damping.

\subsection{Fluid-structure Interaction}

The sound pressure of every point within the sound field can be written as

$$
p(x, y, z, t)=\sum_{i=1}^{n} N_{i}(x, y, z) p_{i}(t)
$$

with $n$ being the number of the nodes in the fluid region, $N_{i}$ being the shape function of the fluid region and $p_{i}$ being the sound pressure. 
The displacement of every element within the structure can be written as,

$$
\mathbf{u}(x, y, z, t)=\sum_{i=1}^{m} \overline{N_{i}}(x, y, z) \mathbf{a}_{i}(t)
$$

with $m$ being the number of the nodes in the structure, $\overline{N_{i}}$ being the shape function of the structure and $\mathbf{a}_{i}$ being the displacement.

Based on the basic equations and boundary conditions of the fluid-structure system, the expression within the fluid region can be written as

$$
\begin{array}{r}
\int_{V_{f}} \delta p\left(\nabla^{2} p-\frac{1}{c_{0}^{2}} \frac{\partial^{2} p}{\partial t^{2}}\right) d V-\int_{S_{b}} \delta p\left(\frac{\partial p}{\partial n_{f}}\right) d S \\
-\int_{S_{f}} \delta p\left(\frac{1}{g} \frac{\partial^{2} p}{\partial t^{2}}+\frac{\partial p}{\partial z}\right) d S \\
-\int_{S_{0}} \delta p\left(\frac{\partial p}{\partial n_{f}}+\rho_{f} \frac{\partial^{2} \mathbf{u}}{\partial t^{2}} \cdot \mathbf{n}_{f}\right) d S=0
\end{array}
$$

The expression of the structure is written as

$$
\begin{array}{r}
\int_{V_{s}} \delta u_{i}\left(\nabla^{2} \sigma+f_{i}-\rho_{s} \frac{\partial^{2} u_{i}}{\partial t^{2}}\right) d V-\int_{S_{\sigma}} \delta u_{i}\left(\sigma n_{s j}-\bar{T}\right) d S \\
-\int_{S_{0}} \delta u_{i}\left(\sigma n_{s j}+p n_{s i}\right) d S=0
\end{array}
$$

with $V_{f}$ and $V_{s}$ being the structure region and fluid region, $S_{0}$ being the interface between fluid and structure, $S_{b}$ being the rigid interface of the fluid, $S_{f}$ being the pressure-released interface of the fluid, and $S_{\sigma}$ being the interface of the structure. Also, subscript $f$ represents fluid, subscript s to represent structure.

Considering the arbitrariness of the $\delta p$ and $\delta u_{i}$, the finite element equation of the fluid-structure system can be obtained,

$$
\left[\begin{array}{cc}
\mathbf{M}_{s} & 0 \\
-\mathbf{Q}^{T} & \mathbf{M}_{f}
\end{array}\right]\left[\begin{array}{c}
\ddot{\mathbf{a}} \\
\ddot{\mathbf{p}}
\end{array}\right]+\left[\begin{array}{cc}
\mathbf{K}_{s} & \frac{1}{\rho_{f}} \mathbf{Q} \\
0 & \mathbf{K}_{f}
\end{array}\right]\left[\begin{array}{l}
\mathbf{a} \\
\mathbf{p}
\end{array}\right]=\left[\begin{array}{c}
\mathbf{F}_{s} \\
0
\end{array}\right]
$$

in which, $\mathbf{p}$ is the pressure vector of the fluid nodes, $\mathbf{a}$ is the displacement vector of the structure nodes, $\mathbf{Q}$ is the fluid-structure coupled matrix, $\mathbf{M}_{f}$ and $\mathbf{K}_{f}$ are the fluid mass matrix and fluid stiffness matrix, $\mathbf{M}_{s}$ and $\mathbf{K}_{s}$ are the structure mass matrix and structure stiffness matrix, $\mathbf{F}_{s}$ is the loading vector on the structure.

\subsection{Boundary conditions}

In this paper, we use PEKERIS waveguide as an example to calculate the sound scattering field of the target. The bottom is represented by an infinite fluid half space, allowing for energy to be transmitted across the water-bottom interface and thereby introducing an additional loss mechanism to the waveguide propagation. It is assumed that the surface and the bottom are plane and parallel such that we can use the integral transform solution technique. The sound speeds are denoted $c_{1}$ and $c_{2}$ for the water and bottom, respectively, and the corresponding densities are $\rho_{1}$ and $\rho_{2}$. Choose a coordinate system with its origin within the waveguide, $\mathrm{z}$ axis vertically upward, and $\mathrm{x}$ axis parallel to the boundaries.

The first boundary condition is that of vanishing pressure at the sea surface,

$$
\left.p(x, y, z)\right|_{z=D}=0
$$

in which, $\mathrm{z}=\mathrm{D}$ means the sea surface.

The remaining boundary conditions are related to the field at the water-bottom interface. They are identical to the boundary conditions used to determine the planewave reflection coefficient, i.e., continuity of particle displacement, and pressure, across the interface. We obtain for continuity of bottom particle displacement,

$$
\left.\frac{1}{\rho_{a}} \frac{\partial p_{a}}{\partial z}\right|_{z=-(H-D)}=\left.\frac{1}{\rho_{b}} \frac{\partial p_{b}}{\partial z}\right|_{z=-(H-D)}
$$

and for continuity of bottom pressure,

$$
\left.p_{a}\right|_{z=-(H-D)}=\left.p_{b}\right|_{z=-(H-D)}
$$

in which, using subscript a to represent fluid in waveguide, subscript $b$ to represent water-bottom and $\mathrm{z}=-(\mathrm{H}-\mathrm{D})$ means water-bottom.

\section{Numerical Simulations}

The geometry is shown in Fig.1. A fluid of constant thickness $\mathrm{H}=10 \mathrm{~m}$ overlies a bottom, which, we assume, is a PEKERIS waveguide for simplicity. An elastic cylindrical shell completely contained within the waveguide is insonified by a plane wave of $1 \mathrm{~Pa}$. We choose a coordinate system with its origin at the centre of the cylindrical shell within the waveguide, $\mathrm{z}$ axis vertically upward, and $\mathrm{x}$ axis parallel to the boundaries. The surface at $\mathrm{z}=\mathrm{D}$ is pressure release and the bottom interface is at $\mathrm{z}=-(\mathrm{H}-\mathrm{D})$.

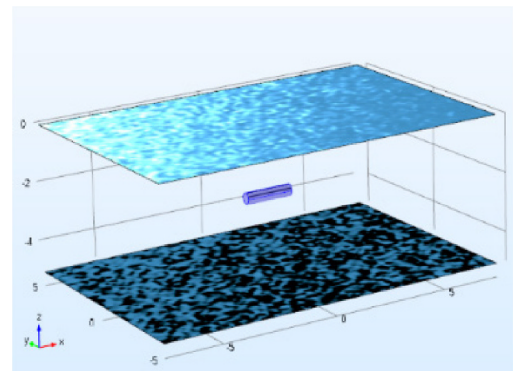

Fig. 1. The geometry of the problem showing a cylindrical shell of radius $0.5 \mathrm{~m}$ and length $2 \mathrm{~m}$ immersed in a shallow water.

The radius of the cylindrical shell (which is at depth $D)$ is $0.5 \mathrm{~m}$, and the length is $2 \mathrm{~m}$, while it is made of Steel AISI 4340, with the Young modulus $E=2.05 \times$ $10^{11} \mathrm{~Pa}$, the density $\rho_{s}=7.85 \times 10^{3} \mathrm{~kg} / \mathrm{m}^{3}$, and the Poisson ratio $\mu=0.28$. Considering the realistic meaning, the physical quantity we discuss here is sound scattering pressure level $(\mathrm{dB})$. 


\subsection{Effects of boundaries}

Sound scattering field of an object in free space and in waveguide are compared together, and to simply the problem, the sea floor is assumed as a hard boundary, on which the sound pressure can be completely reflected.

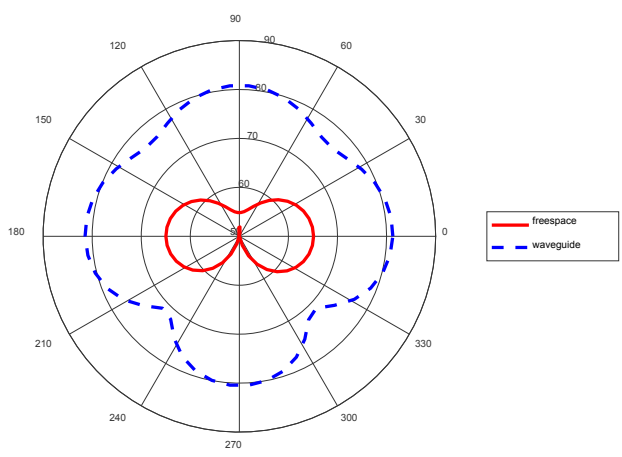

(a) $500 \mathrm{~Hz}$

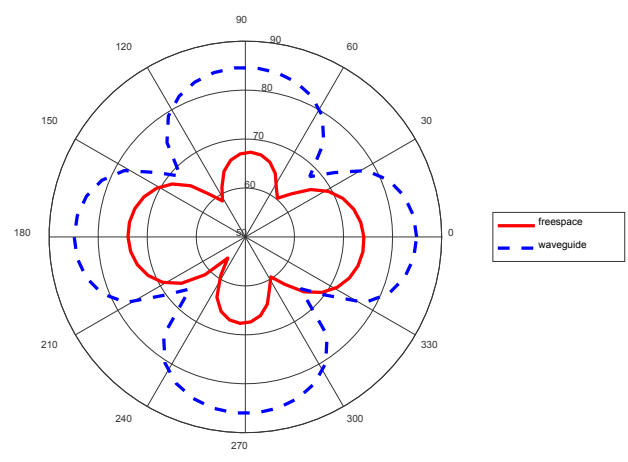

(b) $700 \mathrm{~Hz}$

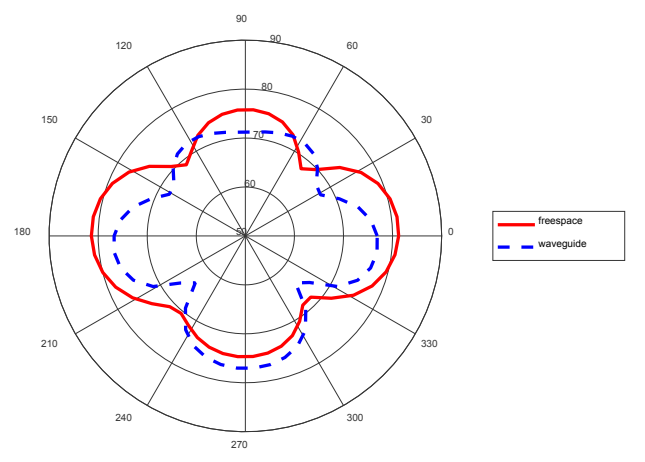

(c) $900 \mathrm{~Hz}$

Fig.2. Contrast between free space and waveguide on $x-y$ plane (far field) (dB).

Fig.2 is about the sound scattering pressure level of an object within free space and the waveguide at different frequency. From Fig.2, we can see that the sound scattering pressure level in waveguide can be about $20 \mathrm{~dB}$ larger than that in the free space at lower frequency, for example, $500 \mathrm{~Hz}$. As the frequency changes from $500 \mathrm{~Hz}$ to $900 \mathrm{~Hz}$, the module value of the object's scattering field in free space changes larger and larger, while changes of the value in waveguide does not have a clear law with the frequency. The difference is variable by the frequency, that is, it gets smaller with the frequency going up. This phenomenon is because the boundaries reflect the sound wave, which makes them intervene and do superposition, while in the free space, the sound wave is emanative, without the superposition of the reflected wave caused by boundaries. Moreover, the number of side lobes is also frequency-related.

\subsection{Effects of incident wave directions}

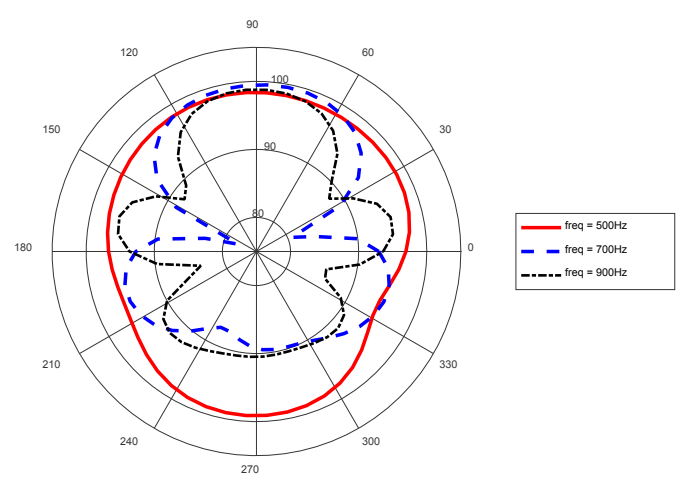

a) Normal to the target (0 degree)

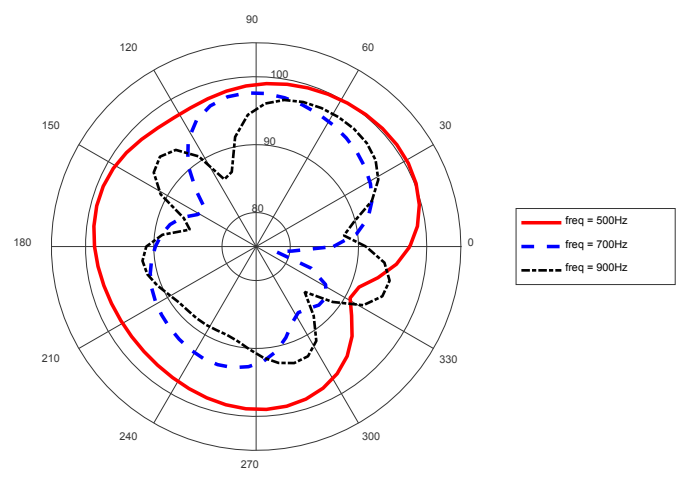

b) 30 degrees to the target

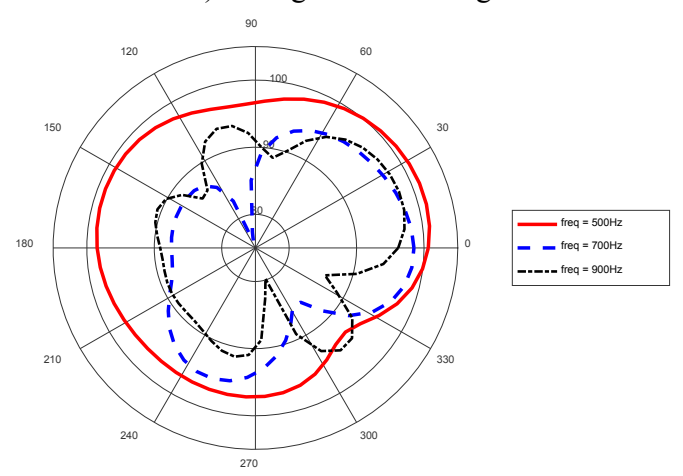

c) 60 degrees to the target

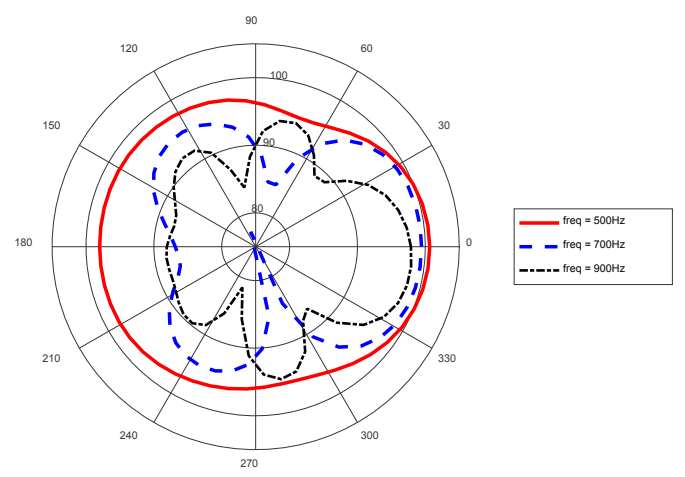

d) End to the target (90 degrees)

Fig. 3. Contrast among different directions of incident wave on $\mathrm{x}-\mathrm{y}$ plane (far field) (dB). 


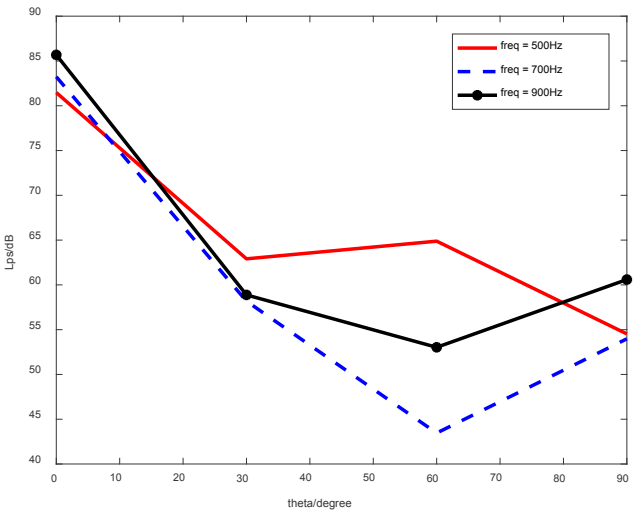

Fig. 4. Contrast between different incident waving directions and frequencies $(\mathrm{dB})$.

In this part, we also do some analyses on $\mathrm{x}-\mathrm{y}$ plane for practical application, that is, the incident wave effect on the sound scattering field of the target in waveguide, and set 4 situations as examples. Firstly, the number of side lobes is still frequency-related. There are few side lobes at $500 \mathrm{~Hz}$ wherever the incident wave is. The amount of side lobes increases as the frequency increases. Secondly, when the incident wave is normal to the object, the distribution of the sound scattering field is axisymmetric about the incident waving direction, which is a law suitable for all the four situations. Thirdly, the change of the incident waving direction cannot change the amplitude of the distribution of the sound scattering field. It just changes the directivity of the sound scattering field. Last, the changes of the scattering pressure are inversely proportional to the frequency, which can demonstrate it is the isolated-mode, not the superposition-modes, that plays an important role in the scattering field at low frequency.

Fig. 4 shows the effect of an incident wave of any direction to the target at different frequency. And 0 degree means the incident waving direction is normal to the target, while 90 degree means the incident waving direction is end to the target. From this figure, we can intuitively see that when the incident wave direction is normal to the target at low frequency, the target strength will be maximum.

\subsection{Effects of seabed impedances}

For this part, use PEKERIS waveguide as the model, and make the impedance change a little, considering the practical situations.

Fig. 5 is to analysis the sound scattering filed of the target at different frequency changing the impedances of the seabed, and we can see that the changes of the seabed impedance make little contribution to the distributions of the sound scattering field. At lower frequency, for example, $500 \mathrm{~Hz}$, we can see the impedance can influence the nodule value, which is in inverse proportion with the impedance. At higher frequency, for example, $900 \mathrm{~Hz}$, the influence of the seabed impedance on the distribution of the sound scattering field is even smaller. Set damping coefficient 0.2 ,

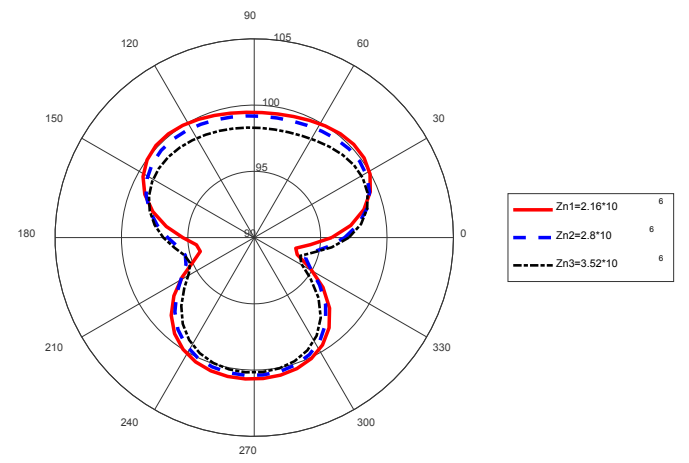

(a) $500 \mathrm{~Hz}$

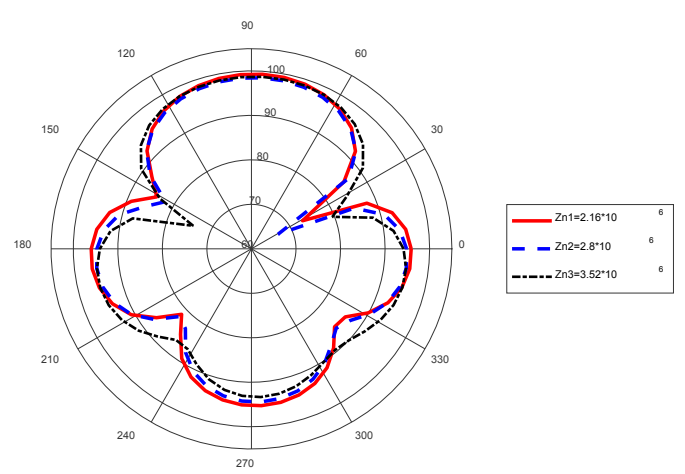

(b) $700 \mathrm{~Hz}$

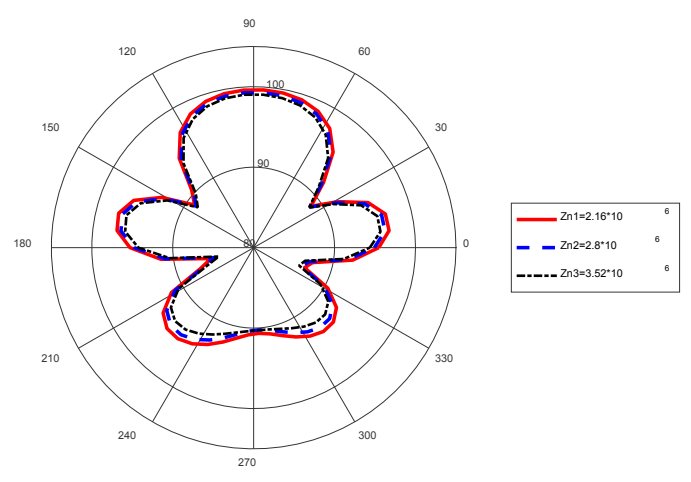

(c) $900 \mathrm{~Hz}$

Fig. 5. Contrast among different seabed impedances on $x-y$ plane (far field) (dB).

\subsection{Effects of target locations}

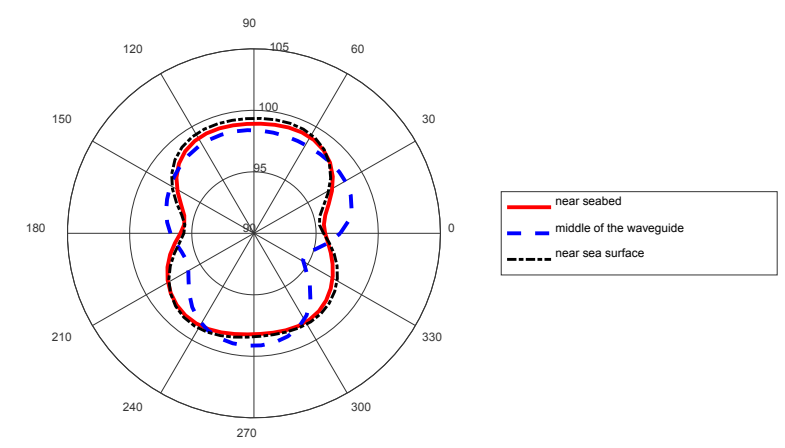

(a) $500 \mathrm{~Hz}$ 


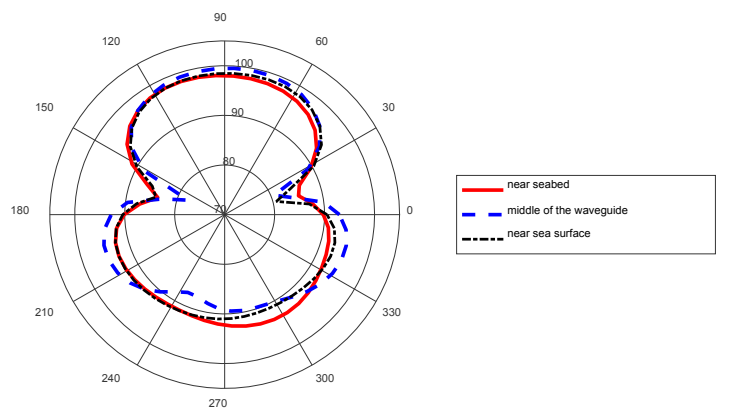

(b) $700 \mathrm{~Hz}$

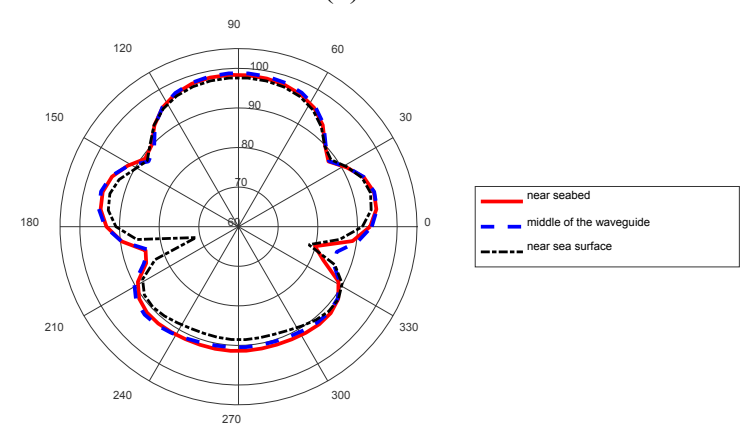

(c) $900 \mathrm{~Hz}$

Fig. 6. Contrast among different target locations on $x-y$ plane (far field) $(\mathrm{dB})$.

Fig.6 is about the effect of the target locations and the sound scattering pressure level of the target is calculated in the figure 6 . It can be easily seen that different locations of the target have a little influence on the scattering field. As the frequency goes up, for example, $900 \mathrm{~Hz}$, changing location will not change the module value of the sound scattering field. When the frequency is lower, the changes of the target location just effect the module value, which doesn't have a clear law among frequency, location and itself.

\section{Conclusions}

According to the analyzation above, we can arrive at some conclusions:

a) The effect of frequency and boundaries on the distribution of the scattering field are stronger than other factors.

b) Boundaries can influence not only the distribution of the scattering field but also the module value at lower frequency, while at higher frequency, the existence of boundaries has more impacts on the distribution.

c) Incident waving directions influence the distribution of the sound scattering field, and have a little impact on the amplitude.

d) The impedances of the seabed have obviously effect on scattering field strength, especially when the frequency is low. The larger the seabed impedance is, the less the module value is.

e) The scattering field strength is also related to the target locations. When the target is in the middle of the waveguide, the module value is the smallest at lower frequency.

The author would like to thank the college of underwater acoustic engineering for the fund. And the work has been also funded by the Natural Science Foundation, No. 11474074.

\section{References}

1. Ingenito F., J.Acoust Soc.Am., 82(6), 2051-2059 (1987)

2. Katsnelson B, Petnikov V, Lynch J., Underwater Acoust., 145-191 (2012)

3. Sammelmann G S, Hackman R H, J. Acoust Soc. Am., 82(1), 324-336 (1987)

4. Ahluwalia D S, Keller J B, Lecture Notes in Phys., 70(12), 14-85 (1977)

5. Varadan V K, Varadan V V. N, Sti/recon Tech. Report N, 80, 103-134 (1979)

6. Jensen F B, Kuperman W A, Porter M B, et al. Computational Ocean Acoustics. (2011)

7. Marburg S, Nolte B, Computional Acoustics of Noise Propagation in Fluids-Finite and Boundary Element Methods. 64-74 (2008) 